Check for updates

Cite this: Chem. Sci., 2019, 10, 2342

๑ All publication charges for this article have been paid for by the Royal Society of Chemistry

Received 8th October 2018

Accepted 21st December 2018

DOI: $10.1039 / \mathrm{c} 8 \mathrm{sc} 04462 \mathrm{~g}$

rsc.li/chemical-science

\section{Simultaneous dual-colour tracking lipid droplets and lysosomes dynamics using a fluorescent probe $\uparrow$}

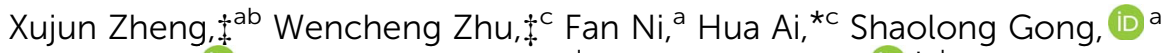 \\ Xiang Zhou, (iD a Jonathan L. Sessler ${ }^{d}$ and Chuluo Yang (D) *ab
}

\begin{abstract}
After entering a cell, most small molecule fluorescent probes are dispersed in the cytoplasm before they then accumulate in a specific organelle or subcellular zone. Molecules that can enter two or more organelles with high selectivity are all but unknown. In this work, we report a naphthalimide-based fluorescent probe, NIM-7, that allows lipid droplets and lysosomes to be labelled simultaneously and with high specificity. These subcellular entities can then be visualized readily through yellow and red fluorescence, using different excitation and detection channels. NIM-7 allows 3D imaging and quantitative visualizing of lipid droplets and lysosomes. It is also able to track simultaneously the movement of lipid droplets and lysosomes in real-time. We also report here that NIM-7 can be used to image both different cell lines and zebrafish embryos.
\end{abstract}

\section{Introduction}

Lipid droplets (LDs) and lysosomes are specialized subunits within cells. These organelles perform specific tasks that are critical to a host of cellular functions. For example, as the most hydrophobic sub-structures within cells, LDs contain a hydrophobic core of neutral lipids (mainly cholesterol and acyl-glycerol) ${ }^{1}$ and serve to store lipids for energy generation and membrane synthesis. Lysosomes are typically acidic organelles (pH 4.5-5.0) that play a critical role in the digestion and clearance of endocytosed and endogenous intracellular materials. ${ }^{2}$ LDs and lysosomes also interact with one another in the context of certain biological processes. For instance, lysosomes are involved in the metabolism of lipid droplets. Moreover, a dynamic cooperation between LDs and lysosomes is critical for cellular energy metabolism with dysfunction being correlated with various metabolic ${ }^{3}$ and lysosomal storage diseases. ${ }^{4}$ An ability to label and track LDs and lysosomes could provide

\footnotetext{
${ }^{a}$ Department of Chemistry, Hubei Key Lab on Organic and Polymeric Optoelectronic Materials, Wuhan University, Wuhan 430072, P. R. China. E-mail: clyang@whu. edu.cn

${ }^{b}$ Shenzhen Key Laboratory of Polymer Science and Technology, College of Materials Science and Engineering, Shenzhen University, Shenzhen, 518060, P. R. China

${ }^{c}$ National Engineering Research Center for Biomaterials, Sichuan University, Chengdu 610064, P. R. China.E-mail: huaai@scu.edu.cn

${ }^{d}$ Center for Supramolecular Chemistry and Catalysis, Shanghai University, Shanghai 200444, P. R. China

$\dagger$ Electronic supplementary information (ESI) available: Experimental details, spectroscopic and cell imaging data, and ${ }^{1} \mathrm{H}$ NMR, ${ }^{13} \mathrm{C}$ NMR, HRMS and HPLC spectra. See DOI: $10.1039 / \mathrm{c} 8 \mathrm{sc} 04462 \mathrm{~g}$

$\ddagger$ These authors contributed equally to this work.
}

new insights into their mutual interactions, as well as their individual roles within working cells. Appropriate probes for this purpose could also see utility in the evaluation of therapeutic agents and the organelle-specific effects of toxins, cellular stress, and disease. Here we report a naphthalimidebased fluorescent probe, NIM-7, that allows lipid droplets and lysosomes to be labelled simultaneously and visualized separately using different excitation and detection channels. Specifically, as a result of the disparate subunits within NIM-7 and the different microenvironments of these two organelles, emission in the yellow and red fluorescence regions is seen for lipid droplets and lysosomes, respectively. This permits quantitative imaging in various cell lines by means of high resolution microscopy.

Owing to their high selectivity, sensitivity, and ease of use, fluorescent probes have assumed central roles within both the chemical and biological communities. ${ }^{5}$ Traditionally, probes were designed to show specific response toward a specific analyte or class of analytes. However, probes capable of displaying different response functions when exposed to different inputs, have attracted increasing attention in recent years, in part because they show promise for the construction of socalled molecular logic gates and molecular keypad lock devices. ${ }^{6}$ Although a considerable number of multi-functional fluorescent probes have been reported to date, as a general rule a differential response is displayed only upon exposure to vastly different species (neutral $v s$. ionic analytes) ${ }^{7}$ or localization in disparate biological environments. ${ }^{8}$ Differential organelle labelling remains a particular challenge. This is mainly due to the fact that small molecule fluorescent probes are generally dispersed in the cytoplasm after penetrating the cell membrane 
before accumulating in a specific organelle or subcellular zone. Although poor probe specificity affords obliquely the possibility of imaging two or more organelles, there are no reports to our knowledge on the use of a single molecule to label and image two or more organelles simultaneously with high selectivity in living cells.

Recently, so-called super-resolution microscopic (SRM) techniques, including STED (stimulated emission depletion), ${ }^{9}$ SIM (structured illumination microscopy), ${ }^{10}$ STORM (stochastic optical reconstruction microscopy) ${ }^{11} /$ PALM (photoactivated localization microscopy), ${ }^{12}$ and RESOLFT (reversible saturatable optically linear fluorescence transitions),${ }^{13}$ have emerged as powerful tools in cell biology due to their resolution beyond the diffraction-limit, axial sectioning capability, as well as high imaging speed. These features have permitted new and exciting insights into the structural organization of cells, as well as the dynamics of biomolecular assemblies over a wide range of timescales..$^{13,14}$ Therefore, combined with an appropriate probe, SRM could permit the concurrent monitoring lipid droplets and lysosomes. The present study was designed to test this possibility.

Inspired by our recent discoveries that a weakly basic derivative of naphthalimide can be protonated within the acidic microenvironment of lysosomes ${ }^{15}$ we were prompted to test further what we expected would be a naphthalimide-based multi-organelle specific fluorescent probe, namely the previously reported system [(E)-2-(2-(dimethylamino)ethyl)-6-(4(diphenylamino)styryl)- $1 H$-benzo[de]isoquinoline-1,3(2H)-dione] (termed NIM-7). ${ }^{16}$ In NIM-7, the diphenylamine, styryl, and 1,8naphthalimide moieties were expected to act as an electron donor (D), a $\pi$-bridge, and an electron acceptor (A), respectively. Predicative photophysical analyses (vide infra) revealed that the emission colour of NIM-7 is not influenced by the acidity. Therefore, protonation of the dimethylamino group of NIM-7, which was expected to drive accumulation of the probe molecules in lysosomes, ${ }^{17}$ was not expected to perturb the inherent red fluorescence. In contrast, the emission characteristics of NIM-7 proved quite sensitive to the polarity of the solvent medium. In particular in hydrophobic environments, such as within LDs, NIM-7 gives rise to a yellow fluorescence. As a result, we postulated that NIM-7 might have a role to play as a twocolour fluorescent probe capable of imaging LDs (or lipid-rich tissue) and lysosomes simultaneously by monitoring the different emission colours produced in these two subcellular environments (Scheme 1). Test of this hypothesis are described below. Briefly, we found that the microenvironment (that is, polarity and acidity) of these two organelles (or tissues) can in fact be distinguished as inferred from cell studies and zebrafish embryos. NIM-7 is able to track LDs and lysosomes dynamics concurrently in real-time. In addition, probe NIM-7 could be used to achieve the super-resolution imaging of LDs and lysosomes at the nanoscale level and at higher resolution than typically seen using laser scanning confocal microscopy (CLSM).

\section{Results and discussion}

NIM-7 was synthesized via the Heck coupling of $N, N$-diphenyl-4vinylaniline with 6-bromo-2-(2-(dimethylamino)ethyl)-1 $H$-benzo

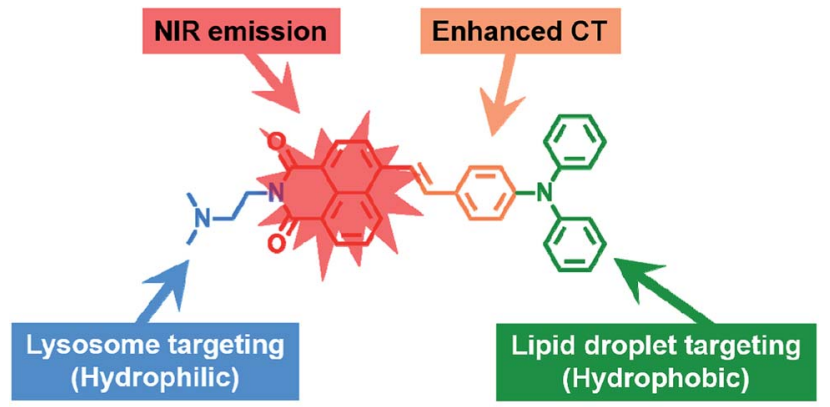

NIM-7

Scheme 1 Molecular structure of the fluorescent probe NIM-7. The function of each moiety within the overall NIM-7 structure is highlighted (CT: charge transfer; NIR: near infrared).

[de]-isoquinoline-1,3(2H)-dione in moderate yield according to the previously reported literature (Scheme S1 $\dagger$ ). ${ }^{16}$ The structure NIM-7 was determined by ${ }^{1} \mathrm{H}-\mathrm{NMR}$ and ${ }^{13} \mathrm{C}-\mathrm{NMR}$ spectroscopies and high-resolution mass spectrometry (HRMS), while the purity was assessed by high performance liquid chromatography (HPLC).

In order to evaluate whether NIM-7 could act as a fluorescent cell imaging probe, we first investigated the chemical stability of NIM-7 in the presence of intracellular nucleophiles, including $\mathrm{GSH}$, Cys, and $\mathrm{H}_{2} \mathrm{O}_{2}$. As shown in Fig. $\mathrm{S} 1, \uparrow$ treatment with an excess of these species cause negligible spectroscopic changes. This leads us to suggest that NIM-7 might prove stable in various subcellular environments. The cytotoxicity and photostablility of NIM-7 in cells were also determined. As shown in Fig. S2a, $\uparrow$ it was found that NIM-7 has no obvious cytotoxicity on four test cell lines (Hep3B, HepG2, HeLa and IMCD3) after $24 \mathrm{~h}$ incubation. This was true even when a 10-fold excess was used relative to what expected to be used for cellular imaging. The photostability of NIM-7 was also tested. Under conditions of CLSM imaging in HeLa cells, $c a$. $90 \%$ of the original fluorescence intensity remained after $15 \mathrm{~min}$ of irradiation (Fig. S2b $\dagger$ ). On the basis of these predicative studies, we considered it worth testing NIM-7 in the context of cellular imaging.

A first set of CLSM imaging studies were carried out using HeLa cells incubated in the presence of NIM-7. As shown in Fig. 1 (first panel), after excitation at $488 \mathrm{~nm}$, the cells were characterized by the presence of dozens of yellow fluorescent spots which nicely colocalized with a commercially available dye (HCS LipidTOX ${ }^{\mathrm{TM}}$ Deep Red Neutral Lipid Stain) for lipid imaging. Upon excitation at $561 \mathrm{~nm}$, numerous red fluorescent spots were observed; again, these were found to colocalize with commercial dye (LysoTracker® Blue DND-22) for lysosome imaging (Fig. 1, second panel). However, these two fluorescent signals do not colocalize with two other organelle-specific dyes, namely MitoTracker ${ }^{\mathrm{TM}}$ Deep Red FM and ER-TrackerTM BlueWhite DPX that are used to probe the mitochondrion and the endoplasmic reticulum, respectively. Moreover, an analysis of the merger of the two excitation outputs (yellow and red) seen for NIM-7 provides support for the contention that the two signals are not located in the same subcellular zone. We thus 


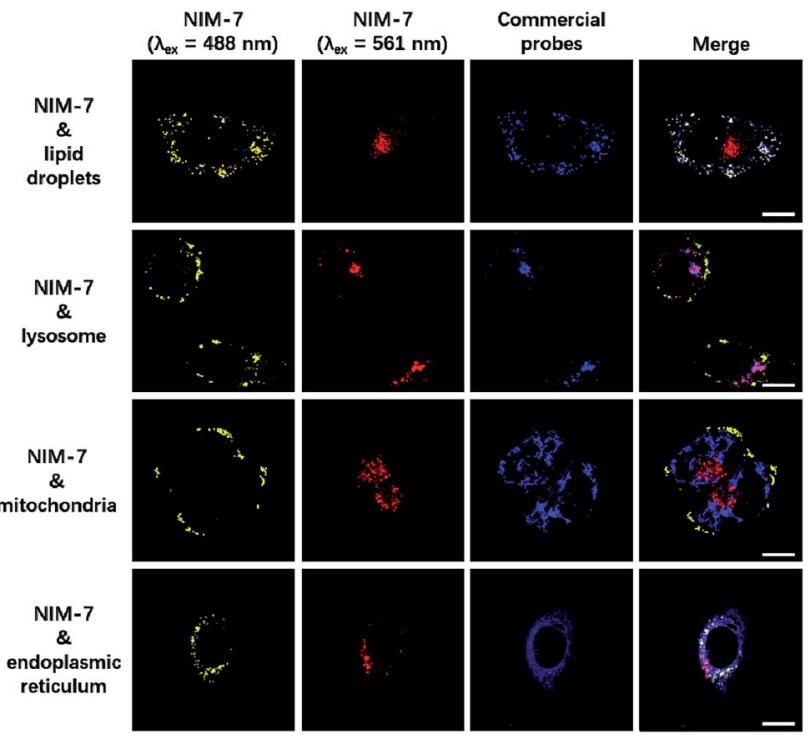

Fig. 1 Confocal microscopic images of HeLa cells stained with NIM-7 and commercial probes. Cells were stained with $0.5 \mu \mathrm{M} \mathrm{NIM-7} \mathrm{for} 1 \mathrm{~h}$, or HCS LipidTOXTM Deep Red Neutral Lipid Stain (1: 1000 dilution) for $0.5 \mathrm{~h}$, or $50 \mathrm{nM}$ LysoTracker® Blue DND-22 for $0.5 \mathrm{~h}$, or $50 \mathrm{nM}$ MitoTracker ${ }^{\mathrm{TM}}$ Deep Red FM for $0.5 \mathrm{~h}$, or 100 nM ER-TrackerTM BlueWhite DPX for $0.5 \mathrm{~h}$. Scale bar: $20 \mu \mathrm{m}$.

infer that the signals are derived from the two targeted organelles, LDs and lysosomes. NIM-7 was also tested in several other cell lines, such as HepG2, Hep3B, and IMCD3. The results, presented in Fig. $\mathrm{S} 3, \uparrow$ are consistent with the notion that NIM-7 can be used as a general probe to visualize simultaneously LDs and lysosomes in a range of cell lines.

To obtain insights into the localization mechanism of NIM-7 in living cells, we disrupted the acidic microenvironment characteristic of lysosomes by adding chloroquine (CQ), which can elevate the $\mathrm{pH}$ of the lysosome from 4.5 to $6.5 .^{15,18}$ After preincubation with $50 \mu \mathrm{M} \mathrm{CQ}$ for $0.5 \mathrm{~h}$, a lower level of NIM-7 accumulation in lysosomes was observed compared to the untreated case (Fig. S4 $\dagger$ ). However, this pretreatment did not influence the LD-based signal produced by NIM-7 (Fig. S4b and $\mathrm{e}^{\dagger}$ ).

It is recognized that LDs are highly dynamic organelles, particularly in the face of an energy shortage. Previous studies have led to an appreciation that autophagy can regulate lipid metabolism under conditions of nutrient deprivation, events that involve the lysosomes. ${ }^{19}$ Several of the authors have also shown that the contents of lipid droplets can be metabolized during glucose starvation..$^{20}$ Therefore, an effort was made to test whether NIM-7 could be used to observe the dynamics of lipid droplet change in cells deprived of glucose. As shown in Fig. $S 5, \dagger$ the number of lipid droplets decreased dramatically after IMCD 3 cells were cultured for $12 \mathrm{~h}$ culture in the absence of glucose. However, no obvious changes on lysosome number were observed. We thus believe that protonation within the acidic microenvironments of lysosomes is the key factor that allows NIM-7 to be used for labelling lysosomes, while the hydrophobicity of NIM-7 permits its use in visualizing hydrophobic LDs.
To explain the imaging colour of NIM-7 in these two organelles, the photophysical behaviour of NIM-7 in solvents with different polarities was investigated. As shown in Fig. 2a and Table S1, $\uparrow$ the photoluminescence (PL) emission spectra of NIM-7 displayed apparent positive solvatochromism; i.e., with increasing solvent polarity, the emission maximum of NIM-7 was red-shifted [e.g., $\lambda_{\text {PLmax }}=549 \mathrm{~nm}$ in toluene; $\lambda_{\text {PLmax }}=$ $670 \mathrm{~nm}$ in dimethyl sulfoxide (DMSO)]. Quantum yields that decreased with polarity were also seen (e.g., $\varphi_{\mathrm{PL}}=0.81 \mathrm{in} \mathrm{Tol}$, $\varphi_{\mathrm{PL}}=0.015$ in DMSO). ${ }^{16}$ In mixtures of Tol + DMSO, intermediate behaviour was seen that was a clear function of the solvent ratio (Fig. S6 $\dagger$ ). Such spectral changes are consistent with strong intramolecular charge transfer (ICT) character in the lowest singlet excited state. However, as can be seen from an inspection of Fig. 2b, the emission wavelength of NIM-7 is insensitive to the $\mathrm{pH}$ in a mixture of $\mathrm{DMSO} / \mathrm{H}_{2} \mathrm{O}(\mathrm{v} / \mathrm{v}, 75 / 25)$.

Taking into consideration that NIM-7 is hydrophobic (Fig. S7 $\dagger$ ), as well as the fact that fetal serum albumin (FSA) is present in the culture medium and that NIM-7 may interact with this and other proteins, we carried out imaging experiments with bovine serum albumin (BSA) either present in, or absent from, the medium. As shown in Fig. S8, $\uparrow$ we found that protein in fact plays an important role in the internalization of NIM-7. In the absence of serum, the localization of NIM-7 in both lipid droplets and lysosomes decreased. However, the expectation that BSA or other proteins would be present under most conditions of use, led us to explore whether NIM-7 could be used to probe the microenvironments of LDs and lysosomes.

To test this possibility, HeLa cells were incubated with NIM7. The fluorescence emission features of the cells were then recorded. As shown in Fig. 3a, the lipid droplet-rich and lysosome-rich zones gave rise to very different emission spectra (Fig. 3b). As noted above, relative to LDs, lysosomes constitute more polar microenvironments characterized by relatively high acidity. These organelles give rise to fluorescence emission features that are similar to those of NIM-7 in DMSO. In contrast, the lipid-rich microenvironments of LDs are reflected in a fluorescence emission spectrum for NIM-7 that resembles that produced in toluene. These differences provide support for the contention that NIM-7 may be used to visualize simultaneously both lipid droplets and lysosomes.

It is well-established that cells are complex threedimensional objects with organelles distributed in different

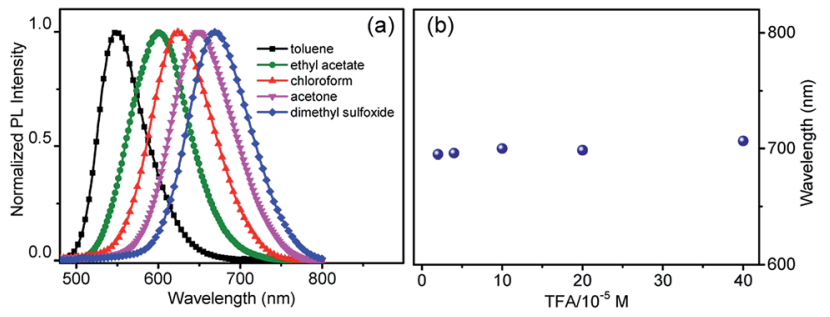

Fig. 2 (a) Normalized fluorescence spectra of NIM-7 recorded in solvents with different polarities under an excitation of $450 \mathrm{~nm}$. (b) Emission maxima recorded for NIM-7 $(20 \mu \mathrm{M})$ upon titration with trifluoroacetic acid (TFA, 20-400 $\mu \mathrm{M})$ in a mixture of $\mathrm{DMSO} / \mathrm{H}_{2} \mathrm{O}(\mathrm{v} / \mathrm{v}$, 75/25). The excitation wavelength was $430 \mathrm{~nm}$. 


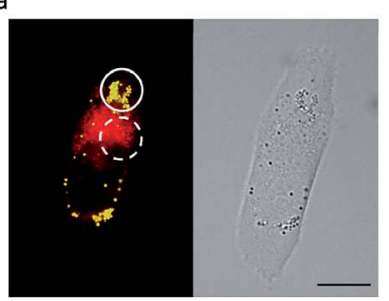

b

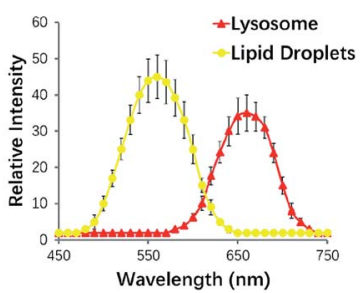

Fig. 3 Fluorescence $\lambda$ scanning results of cells incubated with NIM-7 as recorded over different subcellular zones. (a) Representative image designed to highlight how within the circle the lipid droplet zone was scanned, while within the dotted circle the lysosome zone was scanned. (b) Plot of the mean intensity from scans over various $\lambda$ zones from 450 to $750 \mathrm{~nm}$ with $10 \mathrm{~nm}$ integration. Values are expressed as the mean, $n=5$; the error bars represent the SD; scale bar: $20 \mu \mathrm{m}$.

subcellular zones. CLSM is usually used to capture single layer information upon imaging. CLSM can also be applied to scan different layers of the cells through the $z$ axis to obtain 3D information (Fig. 4a). We thus used CLSM to carry out 3D imaging of LDs and lysosomes in NIM-7 stained HeLa cells. As shown in Fig. 4b, the cellular distribution and numbers of these two organelles were found to be different at each layer along the $z$ axis. From the resulting information, we are able to generate a 3D image of the cells in question (Fig. 4c).

To overcome the diffraction limit associated with conventional fluorescent microscopy and obtain high-resolution images of lysosomes and LDs, we performed super-resolution imaging of NIM-7 in cells using a structured illumination microscope (SIM). Four cell lines were chosen for these studies. The first were HeLa (human cervical cancer) and HepG2 (human liver cancer) cells. Both are human cancer cells. The third and fourth were the IMCD3 (mouse inner medullary collecting duct cell) and TTF (mouse tail-tip fibroblast) cell lines; both are mouse normal cells. Super-resolution imaging of the lysosomes and LDs allowed the structures of these two organelles to be imaged at a higher level than possible using traditional confocal microscopy (Fig. 5a, lower panel). In fact, we could measure quantitatively the number and diameter of the two organelles as established by previous studies. ${ }^{21}$ Compared to the HeLa cells (mean $=408 \mathrm{~nm}$ ), the HepG2 cells $($ mean $=$

a

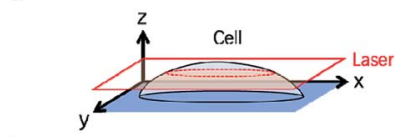

b

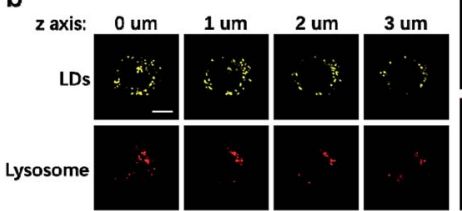

c

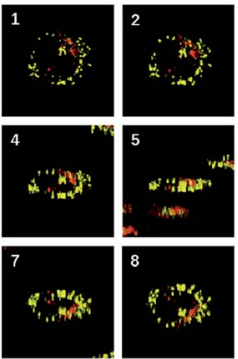

(x axis)

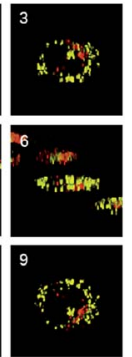

Fig. 4 3D imaging of lipid droplets and lysosomes by NIM-7. (a) A scheme for 3D imaging procedure. (b) The images were captured from different layers. (c) 3D animation of NIM-7 stained cells rotating on $x$ axis. Scale bar: $10 \mu \mathrm{m}$.

$1173 \mathrm{~nm}$ ) are generally contain much larger LDs (Fig. 5b). In the mouse cells, the diameter of the LDs from TTF (mean $=465 \mathrm{~nm}$ ) is smaller than the ones from IMCD3 cells (mean $=135 \mathrm{~nm}$ ). However, TTF cells have many more lysosomes than IMCD3 cells (Fig. 5c). These results suggested that NIM-7 can be used to support super-resolution imaging of LDs and lysosomes with good specificity.

LDs and lysosomes are not static. They typically move within the cytoplasm, presumably to affect more efficiently their dedicated biological functions. To date, only a few reports involving the dynamic tracking lipid droplets ${ }^{20,22}$ or lysosomes $^{15 b, 23}$ have appeared in the literature. Encouraged by the good specificity, high photostability, and low cytotoxicity of NIM-7, it was applied to track concurrently the movement of LDs and lysosomes within HeLa cells. As true in our previous reports, ${ }^{\mathbf{1 5 b}, 20}$ both LDs and lysosomes were seen to move quickly over the course of a short observation period (15 min, Fig. 6). Specifically, during a 45 minute imaging window, over $90 \%$ of these two organelles had moved under normal culture conditions. Of note, the fluorescence intensity did not show an obvious decrease during the imaging time ( $c f$. Fig. S2b $\dagger$ ).

Zebrafish (Danio rerio), have been widely used as a model vertebrate organism. ${ }^{24}$ We thus sought to test if NIM-7 could be used to visualize lysosomes and lipid-rich tissue in living zebrafish. Predicative studies revealed that NIM-7 displays good photostability and little toxicity in zebrafish embryos (Fig. S2 and S9†). Thus zebrafish embryos (3 days post-fertilization, pdf) were labelled with $4 \mu \mathrm{M}$ NIM-7 for $2 \mathrm{~h}$. Under the green channel,
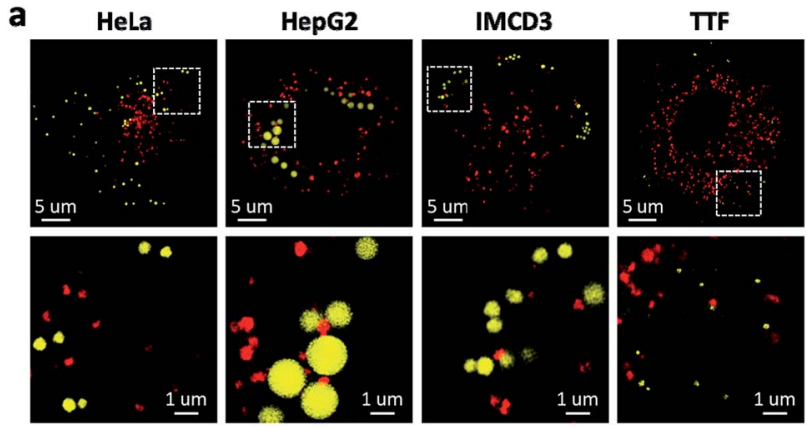

b
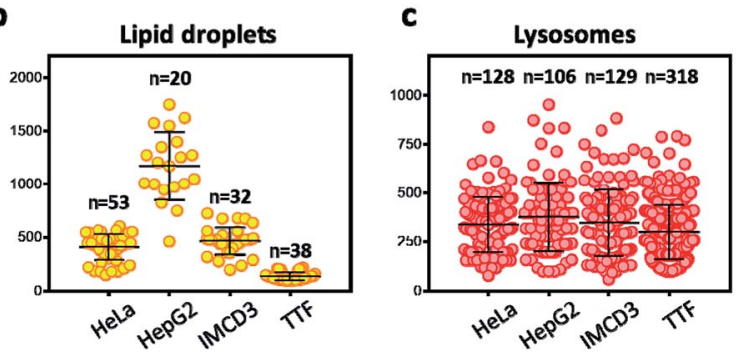

Fig. 5 Super-resolution microscope (structured illumination microscope) imaging and quantitatively measurements of lipid droplets and lysosomes in different types of cells stained with NIM-7 (0.5 $\mu \mathrm{M}, 1 \mathrm{~h})$. (a) Merged picture of the imaged LDs and lysosome in HeLa, HepG2, IMCD3, and TTF cells. Lipid droplets and lysosomes were excited at $488 \mathrm{~nm}$ and $561 \mathrm{~nm}$, respectively. Lower panel: Enlarged view of selected regions shown in upper panel. Total numbers and diameters of LDs (b) and lysosomes (c) visualized by NIM-7 in four cells from (a); error bars represent SD. 
a

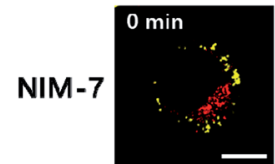

b

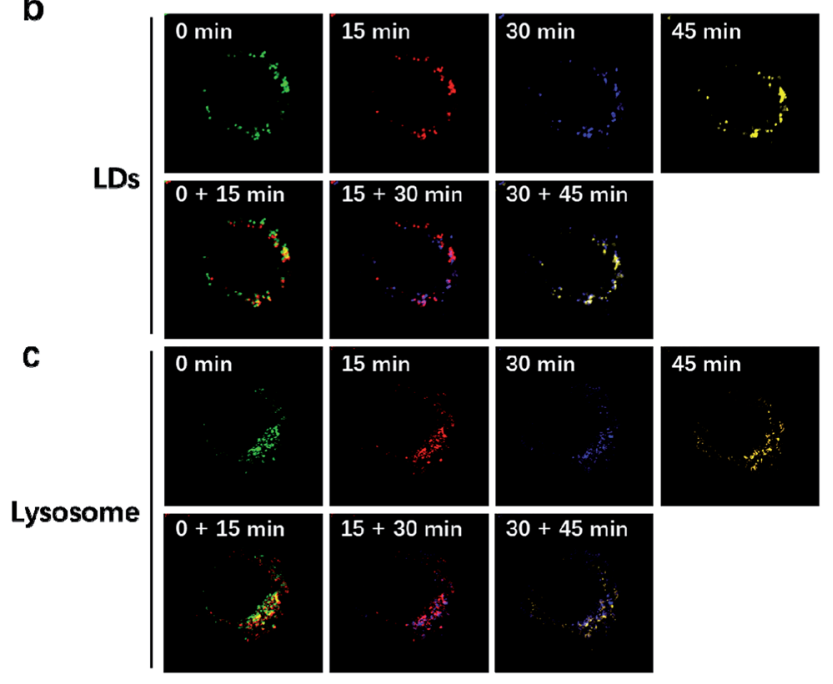

Fig. 6 Real-time tracking lipid droplets and lysosomes dynamics in living cells by NIM-7. (a) Merged picture of the imaged LDs and lysosomes in HeLa cells at different time points. (b) Movement of LDs. (c) Movement of lysosomes. Different pseudo-colours are used to display the movement of lysosomes at different time points. Lipid droplets and lysosomes were excited at $488 \mathrm{~nm}$ and $561 \mathrm{~nm}$, respectively. Scale bar: $10 \mu \mathrm{m}$.

no obvious staining pattern was noted in an overall view (Fig. 7b). However, upon zooming in, a classic lysosome staining pattern could be observed from the lower portion of the eye to the tail of the embryo (Fig. 7d and e). Such a finding provides qualitative support for the suggestion that NIM-7 may be used to visualize lysosomes in vivo. To confirm this inference, we treated the zebrafish embryos with CQ $(200 \mu \mathrm{M})$ for $2 \mathrm{~h}$ followed by NIM-7 staining $(4 \mu \mathrm{M})$ for another $2 \mathrm{~h}$. However, there were no obvious red fluorescent spots observed (Fig. S10 $\dagger$ ). We thus conclude that NIM-7 may be used to visualize lysosomes in zebrafish embryos. We also imaged the stained embryos using a blue channel. Under these imaging conditions, the yolk sac showed yellow fluorescence (Fig. 7c and f). The embryonic yolk zone is well-known to be lipid-rich, thus this result provides support for the notion that NIM-7 may be useful for imaging yolk lipids, as has been seen for other hydrophobic dyes. ${ }^{25}$

\section{Conclusions}

In summary, a fluorescent probe (NIM-7) has been developed that allows for the specific and concurrent imaging of both LDs (or lipid-rich tissue) and lysosomes in cell lines and zebrafish embryos. NIM-7 accumulates in lipid droplets, a microenvironment rich in neutral lipids, where it gives rise to an easy-todiscern yellow fluorescence. NIM-7 also accumulates in lysosomes, a classic acidic microenvironment, where it displays red fluorescence. This probe is able to visualize LDs and lysosomes

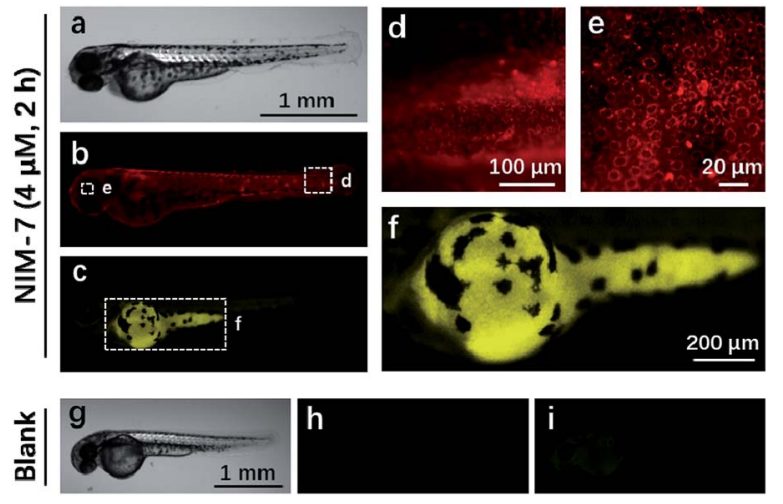

Fig. 7 Zebrafish embryos imaged using NIM-7. The zebrafish embryos ( $3 \mathrm{dpf}$ ) were incubated with $4 \mu \mathrm{M}$ NIM-7 for $2 \mathrm{~h}$. (a) White field image of a zebrafish embryo. Fluorescent images of the zebrafish embryo excited at green channel (505-535 nm) (b) and blue channel (475-495 $\mathrm{nm})(\mathrm{c}) .(\mathrm{d}-\mathrm{f})$ are enlarged from (b) and (c). The images of unlabelled zebrafish embryo, (g) white field; (h) green channel; (i) blue channel.

via super-resolution microscopy. It also allows 3D imaging and real-time tracking using CLSM. To the best of our knowledge, this is the first small-molecule fluorescent probe to stain LDs and lysosomes specifically through different fluorescent emission colours. It and other dual imaging probes may thus emerge as useful tools for quantifying and characterizing various subcellular microenvironments.

\section{Conflicts of interest}

There are no conflicts to declare.

\section{Acknowledgements}

We gratefully acknowledge financial support from the Foundation for Innovative Research Groups of the National Natural Science Foundation (No. 21721005), the Shenzhen Peacock Plan (KQTD20170330110107046), the National Basic Research Program of China (973 Program 2013CB933903), the National Natural Science Foundation of China (No. 91433201 and No. 81621003), and Shanghai University.

\section{Notes and references}

1 (a) R. H. Eckel, K. Alberti, S. M. Grundy and P. Z. Zimmet, J.Lancet, 2010, 375, 181; (b) T. C. Walther and R. V. Farese, Biochim. Biophys. Acta, Mol. Cell Biol. Lipids, 2009, 1791, 459; (c) L. Kuerschner, C. Moessinger and C. Thiele, Traffic, 2008, 9, 338.

2 (a) B. Turk, D. Turk and V. Turk, Biochim. Biophys. Acta, Protein Struct. Mol. Enzymol., 2000, 1477, 98; (b) V. Turk, B. Turk and D. Turk, EMBO J., 2001, 20, 4629.

3 (a) A. H. Mokdad, E. S. Ford, B. A. Bowman, W. H. Dietz, F. Vinicor, V. S. Bales and J. S. Marks, JAMA, 2003, 289, 76; (b) W. Rathmann and G. Giani, Diabetes Care, 2004, 27, 2568; (c) K. F. Adams, A. Schatzkin, T. B. Harris, V. Kipnis, 
T. Mouw, R. Ballard-Barbash, A. Hollenbeck and M. F. Leitzmann, N. Engl. J. Med., 2006, 355, 763.

4 (a) C. Jedeszko and B. F. Sloane, Biol. Chem., 2004, 385, 1017; (b) N. Fehrenbacher and M. Jäättelä, Cancer Res., 2005, 65, 2993.

5 (a) S. Atilgan, T. Ozdemir and E. U. Akkaya, Org. Lett., 2008, 10, 4065; (b) K. C. Ko, J.-S. Wu, H. J. Kim, P. S. Kwon, J. W. Kim, R. A. Bartsch, J. Y. Lee and J. S. Kim, Chem. Commun., 2011, 47, 3165; (c) Z. Guo, S. Nam, S. Park and J. Yoon, Chem. Sci., 2012, 3, 2760; (d) B. Rout, L. Unger, G. Armony, M. A. Iron and D. Margulies, Angew. Chem., Int. Ed., 2012, 51, 12477; (e) M. H. Lee, B. Yoon, J. S. Kim and J. L. Sessler, Chem. Sci., 2013, 4, 4121; (f) A. Atilgan, E. Tanriverdi Eçik, R. Guliyev, T. B. Uyar, S. Erbas-Cakmak and E. U. Akkaya, Angew. Chem., Int. Ed., 2014, 53, 10678; (g) S. Bhuniya, S. Maiti, E. J. Kim, H. Lee, J. L. Sessler, K. S. Hong and J. S. Kim, Angew. Chem., Int. Ed., 2014, 53, 4469; (h) Y. Nissinkorn, N. Lahav-Mankovski, A. Rabinkov, S. Albeck, L. Motiei and D. Margulies, Chem.-Eur. J., 2015, 21, 15981; ( $i$ ) K. Selvakumar, L. Motiei and D. Margulies, $J$. Am. Chem. Soc., 2015, 137, 4892; (j) H. S. Jung, J.-H. Lee, K. Kim, S. Koo, P. Verwilst, J. L. Sessler, C. Kang and J. S. Kim, J. Am. Chem. Soc., 2017, 139, 9972; (k) J. Li, N. Kwon, Y. Jeong, S. Lee, G. Kim and J. Yoon, ACS Appl. Mater. Interfaces, 2018, 10, 12150; (l) Q. Gong, R. Zou, J. Xing, L. Xiang, R. Zhang and A. Wu, Adv. Sci., 2018, 5, 1700664; $(m)$ B. Gu and Q. Zhang, Adv. Sci., 2018, 5, 1700609; (n) X. Xue, S. Jin, Z. Li, C. Zhang, W. Guo, L. Hu, P. C. Wang, J. Zhang and X.-J. Liang, Adv. Sci., 2017, 4, 1700229; (o) L. Yuan, W. Lin, K. Zheng and S. Zhu, Acc. Chem. Res., 2013, 46, 1462; (p) L. Yuan, W. Lin, K. Zheng, L. He and W. Huang, Chem. Soc. Rev., 2013, 42, 622; (q) L. Yuan, W. Lin, S. Zhao, W. Gao, B. Chen, L. He and S. Zhu, J. Am. Chem. Soc., 2012, 134, 13510.

6 (a) O. A. Bozdemir, R. Guliyev, O. Buyukcakir, S. Selcuk, S. Kolemen, G. Gulseren, T. Nalbantoglu, H. Boyaci and E. U. Akkaya, J. Am. Chem. Soc., 2010, 132, 8029; (b) A. P. de Silva and S. Uchiyama, Nat. Nanotechnol., 2007, 2, 399.

7 (a) J. Y. Lee, S. K. Kim, J. H. Jung and J. S. Kim, J. Org. Chem., 2005, 70, 1463; (b) S. Y. Park, J. H. Yoon, C. S. Hong, R. Souane, J. S. Kim, S. E. Matthews and J. Vicens, J. Org. Chem., 2008, 73, 8212; (c) R. Guliyev, S. Ozturk, Z. Kostereli and E. U. Akkaya, Angew. Chem., 2011, 123, 10000; (d) Z. Guo, N. R. Song, J. H. Moon, M. Kim, E. J. Jun, J. Choi, J. Y. Lee, C. W. Bielawski, J. L. Sessler and J. Yoon, J. Am. Chem. Soc., 2012, 134, 17846; (e) M. Ishida, P. Kim, J. Choi, J. Yoon, D. Kim and J. L. Sessler, Chem. Commun., 2013, 49, 6950; (f) Y. Yeon, S. Leem, C. Wagen, V. M. Lynch, S. K. Kim and J. L. Sessler, Org. Lett., 2016, 18, 4396.

8 (a) L. Long, M. Huang, N. Wang, Y. Wu, K. Wang, A. Gong, Z. Zhang and J. L. Sessler, J. Am. Chem. Soc., 2018, 140, 1870; (b) Y. Baek, S. J. Park, X. Zhou, G. Kim, H. M. Kim and J. Yoon, Biosens. Bioelectron., 2016, 86, 885; (c) L. Motiei, Z. Pode, A. Koganitsky and D. Margulies, Angew. Chem., Int. Ed., 2014, 53, 9289; (d) M. H. Lee, J. H. Han, J. H. Lee, N. Park, R. Kumar, C. Kang and J. S. Kim, Angew. Chem., Int. Ed., 2013, 52, 6206; (e) L. Li, X. Shen, Q. H. Xu and S. Q. Yao, Angew. Chem., Int. Ed., 2013, 52, 424; $(f)$ L. Unger-Angel, B. Rout, T. Ilani, M. Eisenstein, L. Motiei and D. Margulies, Chem. Sci., 2015, 6, 5419; $(g)$ Z. Pode, R. Peri-Naor, J. M. Georgeson, T. Ilani, V. Kiss, T. Unger, B. Markus, H. M. Barr, L. Motiei and D. Margulies, Nat. Nanotechnol., 2017, 12, 1161; (h) W. Xu, Z. Zeng, J.-H. Jiang, Y.-T. Chang and L. Yuan, Angew. Chem., Int. Ed., 2016, 55, 13658; ( $i$ ) Y. Liu, J. Niu, W. Wang, Y. Ma and W. Lin, Adv. Sci., 2018, 5, 1700966.

9 S. W. Hell and J. Wichmann, Opt. Lett., 1994, 19, 780.

10 M. G. Gustafsson, J. Microsc., 2000, 198, 82.

11 M. J. Rust, M. Bates and X. Zhuang, Nat. Methods, 2006, 3, 793.

12 E. Betzig, G. H. Patterson, R. Sougrat, O. W. Lindwasser, S. Olenych, J. S. Bonifacino, M. W. Davidson, J. LippincottSchwartz and H. F. Hess, Science, 2006, 313, 1642.

13 S. W. Hell, Science, 2007, 316, 1153.

14 B. Huang, H. Babcock and X. Zhuang, Cell, 2010, 143, 1047. 15 (a) X. Zheng, W. Zhu, D. Liu, H. Ai, Y. Huang and Z. Lu, ACS Appl. Mater. Interfaces, 2014, 6, 7996; (b) W. Zhu, X. Zheng, Y. Huang, Z. Lu and H. Ai, Sci. China: Chem., 2018, 61, 483. 16 H.-H. Lin, Y.-C. Chan, J.-W. Chen and C.-C. Chang, J. Mater. Chem., 2011, 21, 3170.

17 (a) F. K. Fan, S. Nie, D. M. Yang, M. J. Luo, H. Shi and Y. H. Zhang, Bioconjugate Chem., 2012, 23, 1309; (b) X. F. Zhang, C. Wang, Z. Han and Y. Xiao, ACS Appl. Mater. Interfaces, 2014, 6, 21669; (c) M. H. Li, Y. T. Wang, G. J. Liu, H. J. Lu and G. W. Xing, Chin. J. Org. Chem., 2017, 37, 356.

18 F. Yu, Y. Wang, W. C. Zhu, Y. Huang, M. H. Yang, H. Ai and Z. Y. Lu, RSC Adv., 2014, 4, 36849.

19 R. Singh, S. Kaushik, Y. Wang, Y. Xiang, I. Novak, M. Komatsu, K. Tanaka, A. M. Cuervo and M. J. Czaja, Nature, 2009, 458, 1131.

20 X. Zheng, W. Zhu, F. Ni, H. Ai and C. Yang, Sens. Actuators, B, 2018, 255, 3148.

21 (a) R. Perera, S. Stoykova, B. N. Nicolay, K. N. Ross, J. Fitamant, M. Boukhali, J. Lengrand, V. Deshpande, M. K. Selig, C. R. Ferrone, J. Settleman, G. Stephanopoulos, N. J. Dyson, R. Zoncu, S. Ramaswamy, W. Haas and N. Bardeesy, Nature, 2015, 524, 361; (b) R. Allison, J. R. Edgar, G. Pearson, T. Rizo, T. Newton, S. Gunther, F. Berner, J. Hague, J. W. Connell, J. Winkler, J. LippincottSchwartz, C. Beetz, B. Winner and E. Reid, J. Cell Biol., 2017, 216, 1337; (c) C. M. Blouin, S. Le Lay, A. Eberl, H. C. Kofeler, I. C. Guerrera, C. Klein, X. Le Liepvre, F. Lasnier, O. Bourron, J. F. Gautier, P. Ferre, E. Hajduch and I. Dugail, J. Lipid Res., 2010, 51, 945; (d) Y. Y. Chau, R. Bandiera, A. Serrels, O. M. Martinez-Estrada, W. Qing, M. Lee, J. Slight, A. Thornburn, R. Berry, S. McHaffie, R. H. Stimson, B. R. Walker, R. M. Chapuli, A. Schedl and N. Hastie, Nat. Cell Biol., 2014, 16, 367.

22 M. Gao, H. Su, S. Li, Y. Lin, X. Ling, A. Qin and B. Z. Tang, Chem. Commun., 2017, 53, 921.

23 (a) Y. M. Ho, N. P. Au, K. L. Wong, C. T. Chan, W. M. Kwok, G. L. Law, K. K. Tang, W. Y. Wong, C. H. Ma and M. H. Lam, Chem. Commun., 2014, 50, 4161; (b) M. Grossi, 
M. Morgunova, S. Cheung, D. Scholz, E. Conroy, M. Terrile, A. Panarella, J. C. Simpson, W. M. Gallagher and D. F. O'Shea, Nat. Commun., 2016, 7, 10855.

24 (a) G. J. Lieschke and P. D. Currie, Nat. Rev. Genet., 2007, 8, 353; (b) V. E. Fako and D. Y. Furgeson, Adv. Drug Delivery
Rev., 2009, 61, 478; (c) S. K. Ko, X. Chen, J. Yoon and I. Shin, Chem. Soc. Rev., 2011, 40, 2120.

25 (a) K. S. Jones, A. P. Alimov, H. L. Rilo, R. J. Jandacek, L. A. Woollett and W. T. Penberthy, Nutr. Metab., 2008, 5, 23; (b) Y. F. Kang, Y. H. Li, Y. W. Fang, Y. Xu, X. M. Wei and X. B. Yin, Sci. Rep., 2015, 5, 11835. 